\title{
Retos de las ciencias sociales en Nicaragua
}

\section{Luis Serra}

Investigador, Centro de Análisis Socio-Cultural (CASC) UCA

Este artículo plantea algunas reflexiones sobre la pertinencia y los retos que enfrentan las ciencias sociales (en adelante CS) actualmente en nuestro país con el fin de contribuir al debate y al análisis de esta temática que, ciertamente, requiere de un estudio sistemático para dilucidar las tesis planteadas en este ensayo. En este documento se trata de identificar -basado en la información disponible-las principales fortalezas y limitantes que observamos en dos ámbitos básicos del quehacer de las CS -formación e investigación ${ }^{1}-$, así como de plantear los retos que enfrentan en el contexto nacional marcado por la globalización.

Partimos de considerar a las CS como aquellas disciplinas científicas que estudian las actividades que desarrollan los seres humanos. Algunos autores prefieren hablar de "ciencias de la conducta", y en este sentido, las CS han sido definidas como "toda disciplina o rama de la ciencia que aborda la conducta humana en sus aspectos sociales y culturales"2. Entre las disciplinas de larga trayectoria que integran la extensa familia de las CS, y que se han subdividido en distintas especializaciones temáticas, encontramos las siguientes: antropología, economía, psicología, sociología, ciencias políticas, geografía, arqueología, demografía, derecho, educación, geografía humana, comunicación social, historia, trabajo social.

Desde sus inicios en el siglo XIX, las CS han abordado el estudio de la multiplicidad de fenómenos de la realidad social en el contexto de la expansión capitalista moderna, particularmente las problemáticas más acuciantes para los actores sociales en distintos escenarios históricos y espaciales. Hoy día, en un mundo crecientemente globalizado, donde ocurren velozmente los intercambios materiales, de ideas y de poblaciones que impactan la vida humana, se plantea un difícil reto a las CS para poder comprender las prácticas, los discursos, las rupturas del tejido social y las tendencias de cambio.

La relevancia a nivel mundial de las CS en el siglo XXI ha sido destacada por diversos estudios y autores, quienes señalan la necesidad de un conocimiento integral de los graves problemas sociales que aquejan a la humanidad y de propuestas viables para enfrentar flagelos masivos como el hambre, la pobreza, la exclusión, la violencia, el deterioro ecológico, las migraciones. En América Latina, se ha desarrollado históricamente una vinculación teórico-practica entre academia y sujetos sociales que ha permitido a las CS jugar un rol clave en el análisis crítico y propositivo sobre problemáticas que han repercutido en

1 Queda pendiente abordar otros ámbitos de las CS en Nicaragua como la intervención social, la asesoría y acompañamiento de actores sociales, y la incidencia en políticas públicas.

2 Enciclopedia Británica, www.britannica.com, marzo 2008. 
la calidad de vida y en las oportunidades de la gran mayoría de los/las latinoamericanos/ as. Hoy día el compromiso sigue vigente, como lo expresara Atilio Boron "Los cientistas sociales no podemos ser indiferentes viviendo en la región más desigual del mundo, debemos fortalecer el pensamiento crítico y los instrumentos de análisis para aportar a las luchas populares a fin de que los bienes públicos, como la justicia, la educación y la salud, dejen de ser mercancías..."3.

\section{Breve itinerario de las CS en Nicaragua}

En comparación con otros países latinoamericanos, se ha señalado el carácter tardío y parcial de la evolución de las CS en Nicaragua debido en gran parte a la persistencia de un régimen dictatorial que desconfiaba del potencial revelador y cuestionador de las CS. Luego de una primera larga etapa caracterizada por el ensayo periodístico, la crónica histórica y el análisis jurídico de la realidad social de Nicaragua, las CS cobran relevancia durante la segunda mitad del siglo XX en la medida que se expande la educación universitaria. Este periodo de cambios sociales se caracteriza por un rápido crecimiento económico impulsado por la dinámica agroexportadora, las migraciones campo-ciudad, el surgimiento de clases medias y el fortalecimiento del Estado. La apertura de las carreras universitarias de CS en este periodo contribuyó a la institucionalización de la formación profesional ${ }^{4}$, el fomento de la investigación con metodologías científicas y el debate crítico sobre la realidad nacional dentro del marco permitido por el régimen de los Somoza, conforme a la autonomía universitaria y la libertad de cátedra conquistadas en 1958 luego de arduas luchas cívicas.

Este proceso fue apoyado a nivel regional por ofertas de formación universitaria en CS, especialmente en centros universitarios de Costa Rica y México, y por iniciativas de investigación y publicaciones del Consejo Superior de Universidades de Centroamérica (CSUCA), como fue el Programa Centroamericano de Ciencias sociales. Entre los centros de investigación creados en el país se encontraban el Instituto Centroamericano de Administración de Empresas (INCAE), el Centro de Investigaciones y Asesoría SocioEconómica (CINASE), El Instituto de Promoción Humana (INPRUH), El Instituto Histórico Centroamericano (IHCA) y el Centro de Investigaciones de la Realidad nacional (UCA)

Los enfoques dominantes en esos primeros años reflejaban los paradigmas positivistas, funcionalistas y desarrollistas del Norte, como señala Miguel A. Avilés "entre los sociólogos predominaba la tendencia estructural-funcionalista de carácter behaviorista y cuantitativa, y los economistas estaban enmarcados en las tendencias estructurales y desarrollistas de la CEPAL". En el caso de trabajo social, Iris Prado explica que "la formación de trabajadores sociales entre 1961 y 1972 estuvo ligada a una política social caracterizada como restringida, asistencialista y dirigida a la reproducción social con el fin de contribuir a la acumulación de capital y la supeditación de los sectores populares a la clase dominante." ${ }^{6}$ Este enfoque

3 CLACSO (2006) "Herencias, crisis y alternativas al neoliberalismo", panel del Congreso Latinoamericano de Ciencias sociales, Rio de Janeiro.

4 Aunque la carrera de Derecho se impartía desde el siglo XIX en la Universidad de León.

5 AVILÉS, M.A. (1978). "La investigación en las Ciencias sociales en Nicaragua”, Cuadernos de Sociología No4-5, Managua, UCA, p.40

6 PRADO, I. (2003). "La formación de trabajo social en Nicaragua", Encuentro Nº64, 58-74. Managua: UCA Publicaciones, p.60 
de formación profesional se modifica en los 70 con la "reconceptualizacion teóricometodologica" impulsada por el Centro Latinoamericano de Trabajo Social (CELATS).

Igualmente, en otras disciplinas se observa una transformación de los enfoques iniciales, ya que los espacios y los actores universitarios de CS en Nicaragua estaban inmersos en el debate político-ideológico de su tiempo, donde confluían nuevas corrientes latinoamericanas que articulaban la teoría científica con la praxis transformadora: teología de la liberación, educación popular, teoría de la dependencia, investigación-accion-participativa. Estos enfoques críticos de los paradigmas del Norte penetraron rápidamente los espacios de formación e investigación en CS contribuyendo a una toma de conciencia crítica del status quo y un compromiso con el cambio. En 1976 se formó la Asociación Nicaragüense de Cientistas Sociales, la cual realizó su $1^{\circ}$ Congreso de CS en 1977, donde se presentaron y debatieron distintos estudios sobre la realidad nacional en esa coyuntura de crisis del sistema político, destacándose los trabajos realizados por un grupo de cientistas denominado "Pensamiento Crítico".

Luego del derrocamiento de la dictadura, durante el periodo de los 80 las CS se expandieron en distintos ámbitos públicos bajo la promoción y financiamiento estatal que valoraba su rol en el estudio y planificación del cambio social. Como señala Manuel Ortega: "la investigación social conoció un desarrollo inédito en la historia del país...las necesidades de información del nuevo Estado para la transformación del país, y el compromiso de los científicos sociales con esos cambios revolucionarios se convirtieron en fuertes motores para la indagación científica, y al país en un inmenso laboratorio de investigación social"7.

En esta década revolucionaria se observa una multiplicación de estudiantes universitarios en carreras de CS con nuevos planes curriculares, un crecimiento de la demanda laboral de profesionales en distintos entes estatales y organizaciones sociales, una diversificación de centros de investigación en muchos casos articulados en redes regionales como el Consejo Regional de Investigaciones y Estudios Sociales (CRIES), la colaboración de investigadores y docentes de gran experiencia, particularmente latinoamericanos, la producción de estudios científicos sobre diversos temas y la consolidación de ANICS como espacio interdisciplinario donde se debatían temas cruciales de la transición al socialismo en sus congresos y eventos periódicos.

En cuanto a las limitantes en este periodo, se ha señalado el déficit universitario de cientistas sociales, quienes pasaron a desempeñar funciones en el Estado, la conducción vertical y centralista de las universidades bajo el Consejo Nacional de la Educación Superior (CNES), la introducción universitaria de una versión dogmática y obsoleta del materialismo dialéctico e histórico proveniente de la extinta URSS. Por otro lado, la situación de guerra conllevó a la secretividad de la información, la intolerancia al pensamiento crítico y el énfasis en la producción de estudios y planes instrumentales para la supuesta transición al socialismo ${ }^{8}$.

7 ORTEGA HEGG, M. (2000) “La Investigación en Ciencias sociales en Nicaragua”, Consulta sobre Ciencias sociales y Nuevas Prácticas Electrónicas. Cuernavaca, México, p.14

8 CASTILLO PÉREZ, N. (1999). Educación superior: estado y mercado de trabajo 1960-1999. Managua: PAVSA. 
En los 90 cambió el régimen político y el modelo socio-económico, al implementarse un sistema neoliberal basado en el rol protagónico del mercado (gran empresa nacional y extranjera) facilitado por un Estado reducido a su mínima expresión y subordinado al FMI. El soporte universitario de formación e investigación en CS fue minado por la reducción del presupuesto estatal -que implicó la fuga de profesionales, la carencia de equipos y bibliografía- a pesar de la norma constitucional que asignaba un 6\% del presupuesto nacional y de las luchas periódicas de la comunidad universitaria. También se cerraron los centros y programas de investigación social existentes en distintos entes estatales, tales como el Centro de Estudios del Trabajo (Ministerio del Trabajo), el Centro de Investigaciones sobre la Reforma Agraria (Ministerio de Agricultura y Ganadería) y el Centro de investigaciones culturales (Ministerio de Cultura).

Por otro lado, se observó una proliferación de universidades privadas en todo el país enfocadas a la formación de profesionales, pero que soslayaban la investigación científica en un contexto de mercantilización de la educación, con escaso control de calidad y nula planificación de una oferta de formación acorde con las necesidades de desarrollo del país. Al mismo tiempo, se crearon durante los 90 múltiples organizaciones civiles dedicadas al desarrollo social con apoyo de la cooperación externa en las áreas de derechos humanos, agricultura sostenible y medio ambiente, salud preventiva y rehabilitación, crédito y asistencia técnica a pequeñas empresas, equidad de género, generación y étnica, participación ciudadana e incidencia en políticas públicas. El quehacer de estas organizaciones incluia la investigación aplicada a problemas acotados temática y espacialmente, la provisión de algunos servicios a la población empobrecida de las zonas de trabajo, la sensibilización, capacitación y formación de líderes y ciudadanos/as, la intervención social a través de proyectos participativos para el desarrollo de capacidades locales y la incidencia en políticas públicas que promuevan los derechos humanos y la participación ciudadana ${ }^{9}$.

\section{Balance actual de las CS}

En primer lugar, nadie duda hoy día de la pertinencia de las CS en un país que sufre una compleja problemática socioeconómica y política, caracterizada por la pobreza y exclusión, la violencia intrafamiliar y pública, la vulnerabilidad ambiental, la falta y carestía de servicios públicos, el descrédito de las instituciones políticas, la subordinación a instituciones supranacionales, las migraciones y la desintegración familiar, la alta mortalidad infantil y materna, el atraso tecnológico y la baja productividad, la falta de créditos y políticas de estímulo a la pequeña empresa, entre otros problemas. Esta grave y compleja situación que actualmente enfrenta Nicaragua, requiere de estudios científicos de carácter aplicado y propositivo, así mismo los procesos de desarrollo humano requieren de cientistas sociales para el diseño y ejecución eficaz de políticas y proyectos.

En definitiva, en Nicaragua hoy, las CS pueden y deben contribuir al estudio científico, interdisciplinarioyparticipativo deestas graves problemáticas afin dedescribirintegralmente cada problema, de identificar y valorar sus efectos, de explicar los factores condicionantes, 
y por ultimo, de proponer acciones para mitigar, prevenir o superar las raíces del problema. También los cientistas sociales, según su perfil profesional, pueden evaluar la factibilidad de las acciones o planes propuestos, luego elaborar una política, programa o proyecto para someterlo a las instancias de decisión, y posteriormente pueden colaborar en su ejecución, monitoreo y evaluación de forma participativa con las organizaciones ciudadanas.

Una fortaleza clave en las CS del país es que contamos con un amplio grupo de profesionales con sólida formación en distintas disciplinas sociales y con especializaciones de postgrado en universidades del país y del extranjero, con una rica experiencia profesional en las áreas de educación, investigación e intervención social en las distintas etapas que ha vivido el país en las ultimas décadas. Además, hay que reconocer que gran parte de los cientistas sociales han demostrado poseer un compromiso con el desarrollo humano del país.

A.- En el área de FORMACIÓN, encontramos que, a nivel universitario, se ofertan en el país distintas carreras de CS en varias universidades, entre las cuales predominan derecho, psicología, diplomacia y relaciones internacionales, ciencias de la educación y comunicación social. En 2008 se ofertaron 19 carreras de CS a nivel de licenciatura ${ }^{10}$ en 99 sedes universitarias tanto estatales como privadas de distintos departamentos del país, según la información de las 38 universidades que reporta el Consejo Nacional de Universidades (CNU), tal como muestra el siguiente cuadro.

\section{Oferta universitaria de licenciaturas en carreras de CS, 2008}

\begin{tabular}{|l|c|}
\hline LICENCIATURAS & No DE UNIVERSIDADES \\
\hline Derecho * & 30 \\
\hline Psicología & 12 \\
\hline Diplomacia y Relaciones Internacionales & 11 \\
\hline Ciencias de la Educación* (5) y Pedagogía (5) & 10 \\
\hline Economía * & 9 \\
\hline Comunicación Social (5) y Periodismo (3) & 8 \\
\hline Diplomacia y Ciencias Políticas & 5 \\
\hline Sociología * & 4 \\
\hline Trabajo Social & 2 \\
\hline Antropología Social & 1 \\
\hline Historia & 1 \\
\hline Desarrollo Rural & 1 \\
\hline Ciencias sociales Mención en Desarrollo Local & 1 \\
\hline Promoción Social y Humana & 1 \\
\hline
\end{tabular}




\begin{tabular}{|l|c|}
\hline Relaciones Económicas Internacionales & 1 \\
\hline Desarrollo Social & 1 \\
\hline Comunicación Intercultural & 1 \\
\hline $\begin{array}{l}\text { Total de carreras de CS ofertadas en distintos centros } \\
\text { universitarios }\end{array}$ & 99 \\
\hline
\end{tabular}

Fuente: "Inventario de Carreras 2008", Consejo Nacional de Universidades, disponible en: www. cnu.edu.ni

*Nota: Estas carreras contemplan diversas menciones en algunos centros.

Estas cifras muestran que hay una oferta diversificada de formación en CS y que hay una demanda clara de la joven generación nicaragüense en estas carreras. Sin embargo, se observa una concentración en las primeras seis disciplinas del cuadro, particularmente en derecho, con un tercio del total, a pesar de la saturación observada en el mercado laboral.

Según los datos disponibles, la evolución de la matrícula estudiantil ha diferido en las distintas carreras de CS, por ejemplo en la UNAN Managua se observa un crecimiento sostenido en antropología social que pasó de 25 matriculados en 1996 a 123 en 2002 y a 167 en 2005. Igualmente, en ciencias políticas se incrementó la matrícula de 58 en 2001 a 241 en 2005 . Por el contrario, se observa un descenso en la carrera de historia de 146 estudiantes en 1996 a 94 en 2000 y a 15 en $2005^{11}$. En el caso de la UCA, el descenso de la matrícula a inicios de los 90 en las carreras de sociología y trabajo social llevó en 1996 a una fusión parcial de ambas al crear un tronco común, y posteriormente, en 2002 se cerró el turno diurno y se trasladó a la modalidad sabatina por encuentros. Este cambio de modalidad observado en muchas carreras de CS abrió las puertas a un nuevo tipo de estudiante universitario: gente adulta que trabaja durante la semana, que reside en el interior del país y que tiene otros estudios, inclusive universitarios, pero siente la necesidad laboral y personal de una formación en CS. Hay que destacar que en las carreras de CS nos encontramos con una joven generación de estudiantes que, en su mayoría, posee una sensibilidad social, vocación profesional e interés de contribuir al desarrollo del país.

Por otro lado, las universidades ofrecen una variedad de cursos de postgrado en CS, principalmente cursos cortos de actualización profesional y diplomados de especialización. A nivel de maestrías en CS encontramos en 2008 una oferta de 27 programas, solamente en las 10 universidades pertenecientes al CNU, según nos muestra el siguiente listado:

10 En varias carreras de Licenciatura se ofrece un título intermedio de diplomado o técnico superior.

11 CASTILLO, M. \& ORTEGA, M. (2007) "La situación de las Ciencias sociales en la UNAN Managua", en RAMOS, C. Las Ciencias sociales y La Educación Superior Pública en Centroamérica, El Salvador: FLACSO. 


\section{Programas de maestría en CS, 2008 - universidades del CNU}

\begin{tabular}{|c|c|}
\hline $\begin{array}{l}\text { Universidad Cen- } \\
\text { troamericana (UCA) }\end{array}$ & $\begin{array}{ll}\text { - } & \text { Maestría en Desarrollo Local y Descentralización }\left(2^{\mathrm{a}}\right. \\
\text { edición) } \\
\text { - } \\
\text { Maestría en Administración y Dirección de Empresas }\left(24^{\mathrm{a}}\right. \\
\text { ed.) } \\
\text { - Maestría en Perspectivas de Género y Desarrollo ( } 2^{\mathrm{a}} \text { ed.) } \\
\text { Maestría en Políticas y Derechos de Niños/as y Adolescen- } \\
\text { - } \quad \text { Maes }\left(2^{\mathrm{a}}\right) \\
\text { - }\end{array}$ \\
\hline $\begin{array}{l}\text { Universidad Nacional } \\
\text { Autónoma de León } \\
\text { (UNAN) }\end{array}$ & $\begin{array}{l}\text { - Maestría en Turismo y Desarrollo } \\
\text { - Maestría Psicología Clínica y de la salud } \\
\text { - Maestría en Salud Ocupacional } \\
\text { - Maestría en Educación Rural } \\
\text { - Maestría Actualización de Programas de Inglés } \\
\text { - Maestría en Integración Regional }\end{array}$ \\
\hline $\begin{array}{c}\text { Universidad Autónoma } \\
\text { de Managua (UNAN) }\end{array}$ & $\begin{array}{ll}\text { - } & \text { Maestría en Pedagogía con Mención en Docencia Universi- } \\
\text { - } & \text { Maria } \\
\text { - } & \text { Maestría en Didáctica Especial de la Lengua y la Litera- } \\
\text { - } & \text { Maestría en Adminología Hispánica ( } 3^{a} \text { ed.) } \\
\text { - } & \text { Maestría en Estudios Históricos Latinoamericanos y del } \\
\text { - } & \text { Maestría en Economía Publica y del Desarrollo } \\
\text { - } & \text { Maestría en Economía Agrícola } \\
\text { - } & \text { Maestría en Derecho Empresarial } \\
\text { - } & \text { Maestría en Economía de la Salud }\end{array}$ \\
\hline $\begin{array}{l}\text { Universidad Politécnica } \\
\text { (UPOLI) }\end{array}$ & $\begin{array}{ll}- & \text { Maestría en Derecho Económico. (2a ed.) } \\
\text { - } & \text { Maestría en Derecho Laboral Empresarial. ( } 2^{\mathrm{a}} \text { ed.) } \\
\text { - } & \text { Maestría en Derecho Procesal. (6 } 6^{\mathrm{a}} \text { ed.) } \\
\text { - } & \text { Maestría en Derecho Mercantil (2 } 2^{\mathrm{a}} \text { ed.) } \\
& \text { Maestría en Derecho Penal y Procesal Penal. (6 } 6^{\mathrm{a}} \text { ed.) }\end{array}$ \\
\hline $\begin{array}{c}\text { Universidad de las } \\
\text { Regiones Autónomas de } \\
\text { la Costa Caribe Nica- } \\
\text { ragüense (URACCAN) }\end{array}$ & - Maestría en Salud Intercultural \\
\hline
\end{tabular}

Fuentes: Páginas Web de las universidades del CNU, marzo 2008. 
Parte de estos programas de postgrado ha surgido a partir de convenios de cooperación con universidades de otros países, aprovechando así una valiosa expertise académica internacional, además de fondos para bibliografía, equipos y becas a estudiantes. Paralelamente, los graduados nicaragüenses se han beneficiado de programas regionales de formación postgraduada en CS implementados por FLACSO y universidades de la región, además de las oportunidades que se brindan en modalidad virtual por CLACSO y en modalidad presencial por universidades de Costa Rica, México, Brasil, USA y Europa.

En cuanto a las debilidades de la formación universitaria en CS podemos señalar la carencia de becas para estudiantes de escasos recursos y el encarecimiento de la educación universitaria (aranceles, textos y materiales, transporte, comida y alojamiento) frente a un empobrecimiento de la población; muchos estudiantes con débil formación en secundaria y reducido acceso a Internet; una planta docente de tipo horario con escaso tiempo y recursos para investigación; carencia de programas de formación con enfoques multidisciplinarios, débil integración entre la teoría-practica, entre la universidad y los actores sociales acuciados por una problemática compleja.

B) En el área de INVESTIGACIÓN en CS se observan diversos actores laborando: universidades, centros de estudio, organizaciones de desarrollo, instituciones estatales, empresas de consultoría, organismos de cooperación e instituciones multilaterales. Existe en el país una serie de centros de investigación que realizan estudios sobre diversas temáticas, divulgan los resultados a través de foros, conferencias, publicaciones y cursos, cuentan con centros de documentación especializados con creciente acceso virtual, existen redes y coordinaciones entre centros de investigación destacándose CLACSO a nivel latinoamericano y FLACSO a nivel centroamericano.

Se destaca la labor investigativa en CS que realizan las universidades y centros adscritos, donde se organizan concursos y jornadas de investigación para la discusión de sus trabajos. La producción de estudios sociales monográficos de graduación en pregrado y postgrado muestra una riqueza y variedad que hasta la fecha no ha sido divulgada, ni tomados en cuenta sus planteamientos. Gran parte de la investigación de Nicaragua es producida en las 10 universidades miembros del CNU donde funcionan actualmente 22 Centros de investigación en CS, según muestra el siguiente cuadro.

\section{Centros de investigación en CS de universidades CNU - 2008}

\begin{tabular}{|l|l|}
\hline & $\begin{array}{l}\text { Instituto de Estudio y Promoción de la Autonomía. } \\
\text { Instituto de Promoción, Investigación Lingüística y Rescate Cultural. } \\
\text { Centro de Estudios Socio Ambientales } \\
\text { Instituto de Medicina Tradicional y Desarrollo Comunitario } \\
\text { Instituto de Recursos Naturales, Medio Ambiente y Desarrollo Sos- } \\
\text { tenible }\end{array}$ \\
\hline
\end{tabular}




\begin{tabular}{|c|l|}
\hline $\begin{array}{c}\text { UNAN MANA- } \\
\text { GUA }\end{array}$ & $\begin{array}{l}\text { Instituto nicaragüense de Investigaciones Económicas y Sociales } \\
\text { Centro de Investigaciones y Estudios de la Salud } \\
\text { Centro de Investigaciones Socio-Educativas } \\
\text { Centro de Investigaciones Económicas y Tecnológicas }\end{array}$ \\
\hline \multirow{3}{*}{ UPOLI } & $\begin{array}{l}\text { Instituto de Desarrollo Humano. } \\
\text { Centro de Investigación Latinoamericano y el Caribe } \\
\text { Instituto de Capacitación e Investigación en Desarrollo Rural Inte- } \\
\text { gral } \\
\text { Instituto Martin Luther King } \\
\text { Centro de Investigación y Educación }\end{array}$ \\
\hline BICU & $\begin{array}{l}\text { Centro de Investigaciones Económicas } \\
\text { Centro de Investigación y Documentación de la Costa Atlántica } \\
\text { Programa de Capacitación y Desarrollo }\end{array}$ \\
\hline UCA & $\begin{array}{l}\text { Instituto de Educación de la UCA } \\
\text { Centro de Análisis Socio-Cultural } \\
\text { Programa Interdisciplinario de Estudios de Genero } \\
\text { Instituto de Historia de Nicaragua y Centroamérica } \\
\text { Instituto de Investigación y Desarrollo "Nitlapan" }\end{array}$ \\
\hline
\end{tabular}

Fuente: Consejo Nacional de Universidades, “Centros de investigación”, disponible en: www. cnu.org.ni, marzo 2008.

Además de estos centros, habría que incluir las investigaciones que realizan los departamentos académicos de las universidades a través de sus docentes y estudiantes. Por otro lado, existen varias organizaciones civiles de desarrollo que han realizado investigaciones sobre temáticas socio-políticas, entre las cuales se destacan el Instituto de Promoción de la Democracia (IPADE), el Centro de Comunicación (CINCO), el Centro de Derechos Constitucionales (CDC), la Fundacion Desafíos, la Fundacion para el Desafío Global (FIDEG), el Instituto de Estudios Estratégicos y políticas Publicas (IEEPP).

Diversos organismos multilaterales (PNUD, UNFPA, UNICEF, OIT, BM, BID) han impulsado importantes estudios en Nicaragua y en la región sobre temáticas sociales de interés, destacándose por su rigor y relevancia los Informes de Desarrollo Humano del PNUD y los estudios sobre la dinámica de población del UNFPA. También hay que mencionar la producción individual de destacados investigadores como Alejandro Serrano, Carlos Tunnerman, Oscar R. Vargas. La divulgación de los resultados de estudios se realiza generalmente de forma escrita y verbal en foros y seminarios realizados en el país, así como en congresos organizados a nivel regional por las asociaciones de antropología, ciencias políticas, historia, trabajo social, sociología.

Entre las limitantes en investigación social se ha señalado la reducción de fondos tanto de fuentes estatales como de cooperación externa, la escasa coordinación entre los centros de investigación y de éstos con las instancias de formación. Gran parte de los estudios 
sociales se realiza por necesidad financiera, como consultorías de corto plazo con métodos predeterminados, fines instrumentales y difusión restringida. Esto transforma a los centros de investigación en proveedores de servicios según la demanda del mercado, relegando sus fines de análisis crítico y aporte al desarrollo nacional ${ }^{12}$.

Un obstáculo evidente es la falta de acceso a bases de datos e información producida por instituciones públicas, así como a estudios realizados por empresas de consultoría bajo contrato que establece su confidencialidad. La debilidad teórica de muchos estudios ha sido señalada por Manuel Ortega: "predomina un neopositivismo que sigue creyendo que los datos hablan por sí mismos y un pragmatismo inclemente que sigue afirmando que la única investigación valiosa es aquella que produce resultados útiles y visibles, no importando su validez ética ni científica" ${ }^{13}$. Por otro lado, la carencia de enfoques interdisciplinarios conduce a visiones parciales o "reduccionistas" de los temas estudiados, tal como resulta de abordajes meramente económicos o jurídicos. Otra limitante señalada es que existe un flujo de investigadores de otros países que realizan estudios sociales en Nicaragua que no se traducen al español ni se difunden en el ámbito nacional.

\section{Retos para las ciencias sociales}

Para responder a los desafíos que plantea la grave problemática social de Nicaragua, las CS deben abordar seriamente los retos observados a nivel interno, es decir, plantearse la superación de las limitantes en las áreas de formación e investigación potenciando nuestras capacidades y aprovechando las oportunidades que ofrece el contexto regional. Gran parte de estos retos son comunes a las CS de la región centroamericana según muestra un estudio reciente hecho por FLACSO El Salvador ${ }^{14}$, entre los cuales destacamos en Nicaragua los siguientes:

- Debemos actualizar los planes curriculares de formación en CS incorporando las competencias necesarias para desempeñarse en mercados laborales globalizados, la promoción del pensamiento crítico-propositivo y una sólida ética social. Es preciso articular el aprendizaje teórico y práctico a través de talleres, prácticas y pasantías.

- También se debe mejorar las estrategias de mercadeo de los programas en CS para aumentar la matrícula en aquellas carreras en disminución, complementadas con políticas de retención y acompañamiento de los nuevos estudiantes.

- Promover la investigación entre los docentes universitarios de CS y brindarles oportunidades de actualización profesional sistemática e interdisciplinaria, incluyendo enfoques pedagógicos centrados en el aprendizaje y manejo de espacios virtuales.

- Dado el carácter multicausal de los problemas sociales, es preciso implementar programas de investigación multidisciplinarios y de largo plazo -con enfoques de género, generación y etnia- basados en métodos tanto cuantitativos como cualitativos, tratando de articular los niveles local-nacional-global de la problemática analizada.

12 BEBBINGTON, A. (ed.) Investigación y cambio social: desafíos para las ONG en Centroamérica y Mexico. Guatemala: Ed. Ciencias sociales.

13 ORTEGA HEGG, M. (2000) op.cit. p.9

14 RAMOS, C. (2007) Las Ciencias sociales y La Educación Superior Pública en Centroamérica, El Salvador: FLACSO. 
- Además de estudios empíricos aplicados a problemas sociales, las CS deben producir conceptos y teorías propios y pertinentes en el contexto de América Latina, evitando la adopción acrítica de teorías generadas en y para otras sociedades. Como dijo B. de Souza "debemos retomar los retos clásicos y generales de las ciencias sociales, pero desde América Latina"15

- Es preciso ampliar los espacios de comunicación interdisciplinaria y debate científico entre profesionales de las CS sobre la realidad nacional y regional, así como la publicación de resultados de investigación aprovechando las nuevas tecnologías de la información. Hay que ampliar el acceso a bases de datos e información, en especial de instituciones estatales que deben acatar la nueva ley de acceso a información pública.

- Crear o fortalecer las asociaciones gremiales de cientistas sociales y su vinculación con organizaciones homólogas de otros países. Al mismo tiempo, es preciso desarrollar un espacio amplio interdisciplinario en CS como ha sido ANICS. Paralelamente, esas asociaciones debieran articularse con los sujetos sociales y políticos que trabajan por superar la problemática socio-económica del país para aportar sus competencias profesionales.

- En las ofertas de trabajos en el área de CS, hay que tener en cuenta las competencias específicas (perfil de egreso) de las distintas disciplinas a fin de evitar el sesgo favorable a ciertas carreras tradicionales y la discriminación de otras, así como la inequidad de oportunidades por razones de género, generación o etnia.

- Frente al condicionamiento externo de las CS, el economista y presidente de Ecuador, F. Correa señaló recientemente que "es necesario recuperar la autonomía de la producción investigativa y de las agendas respecto a las fuentes de financiamiento, y la soberanía de la oferta de programas de formación en función de criterios académicos y de necesidades sociales de nuestros países"16.

En conclusión, las CS han logrado posicionarse en el mundo académico y político del país gracias a sus aportes indudables al análisis de las problemáticas sociales y a la formulación de políticas y programas sociales. Sin embargo, quedan pendientes fuertes desafíos para fortalecer la formación y la investigación en CS a fin de contribuir eficazmente al desarrollo humano y sostenible de nuestra sociedad en el marco regional y global. 